\title{
FORMATION OF PLUMES IN THE HEAD-ON COLLISIONS OF GALAXIES
}

\author{
VLADIMIR KORCHAGIN \\ Institute of Physics, Stachki 194, Rostov-on-Don, Russia \\ TOSHIO TSUCHIYA \\ Department of Astronomy, Faculty of Science, \\ Kyoto University, Kyoto 606-01,Japan \\ AND \\ KEIICHI WADA \\ National Astronomical Observatory, Mitaka, Tokyo 181, Japan
}

We have studied the collisional interaction of disk galaxies using SPH and $\mathrm{N}$-body numerical simulations. We follow the dynamics of a two-component star-gas disk centrifugally balanced by the gravity of a rigid halo. A companion galaxy is modeled self-consistently, and its two-component disk is balanced by the potential of the stellar halo. This model allows us to follow the dynamics of the gas component in the colliding galaxies simultaneously with the dynamics of the collisionless halo of the companion.

The specific goal of our paper was to study the formation of plumes in the head-on collisions of galaxies. Our results can be summarized as follows: 1. We found that the formation of a ring in the disk of a primary does not depend essentially on the admixture of gas. On the contrary, the presence of gas in the intruder is crucial for the formation of plumes connecting two interacting galaxies. A low-mass intruder with the gas mass about a few percent of the primary's gas content forms a well developed plume.

2. In agreement with previous studies we found that the amplitude of the outwardly propagating ring strongly depends on the relative mass of the intruder galaxy. The intruder galaxy with mass equal to $10 \%$ of the mass of the primary does not form any noticeable ring structure in the disk of the primary, and the bridge becomes the main "fingerprint" of the interaction. 3. Finally we demonstrated that a direct collision forms a bridge of stars stripped from the stellar halo of the companion. The fate of the halo of an intruder depends on its mass. Most of the stars of a low-mass intruder are dispersed after the collision, but the central cores of the halos of higher mass intruders survive the collision. 